PROCEEDINGS OF THE

AMERICAN MATHEMATICAL SOCIETY

Volume 130, Number 5, Pages 1275-1277

S 0002-9939(01)06255-4

Article electronically published on October 5, 2001

\title{
ON THE CLASS NUMBER OF CERTAIN IMAGINARY QUADRATIC FIELDS
}

\author{
J. H. E. COHN
}

(Communicated by David E. Rohrlich)

\begin{abstract}
Theorem. Let $n>2$ denote an integer, $D$ the square-free part of $2^{n}-1$ and $h$ the class number of the field $Q[\sqrt{-D}]$. Then except for the case $n=6, n-2$ divides $h$.
\end{abstract}

Theorem. Let $n>2$ denote an integer, $D$ the square-free part of $2^{n}-1$ and $h$ the class number of the field $Q[\sqrt{-D}]$. Then except for the case $n=6, n-2$ divides $h$.

This generalises Theorem 5.3 of [2], which derives the same conclusion under the restrictions that $n-2$ be squarefree and coprime to 6 , and provides a new proof of the result in [1] that for each $g$ there are infinitely many imaginary quadratic fields whose class number is divisible by $g$.

Proof. Here the Diophantine Equation $2^{n}-1=D a^{2}$ has at least one solution, with $a$ odd and $D \equiv 7(\bmod 8)$; in particular $D \geq 7$ and so the only units in the field are \pm 1 . Thus in the field we obtain $\left(\frac{1}{2}(1+a \sqrt{-D})\right)\left(\frac{1}{2}(1-a \sqrt{-D})\right)=2^{n-2}$ where the ideal $\left[\frac{1}{2}(1+a \sqrt{-D})\right]$ and its conjugate are coprime; thus $\left[\frac{1}{2}(1+a \sqrt{-D})\right]=\pi^{n-2}$ for an ideal $\pi$ having norm 2 . Let $\lambda=(h, n-2)$ with $h=\lambda \mu, n-2=\lambda \nu$ and $(\mu, \nu)=1$. Since the ideal $\pi^{h}$ is principal, it follows that $\left[\frac{1}{2}(1+a \sqrt{-D})\right]^{\mu}=\pi^{\lambda \mu \nu}=\left(\pi^{h}\right)^{\nu}=[\delta]^{\nu}$ for some algebraic integer $\delta$ in the field, and so $\left(\frac{1}{2}(1+a \sqrt{-D})\right)^{\mu}= \pm \delta^{\nu}$. In view of $(\mu, \nu)=1$, it then follows that $\frac{1}{2}(1+a \sqrt{-D})= \pm \gamma^{\nu}$ for some other algebraic integer in the field, $\gamma$. It merely remains to show that $\nu=1$, for then $n-2=\lambda \mid \lambda \mu=h$.

We show first that $\nu$ has no odd prime factor $p$, for otherwise we should find, absorbing the \pm sign into the right-hand side, that for some odd rational integers $\alpha$ and $\beta, \frac{1}{2}(1+a \sqrt{-D})=\left(\frac{1}{2}(\alpha+\beta \sqrt{-D})\right)^{p}$, and then equating real parts gives

$$
2^{p-1}=\alpha \sum_{r=0}^{\frac{1}{2}(p-1)}\left(\begin{array}{c}
p \\
2 r
\end{array}\right) \alpha^{p-2 r-1}\left(-D \beta^{2}\right)^{r} .
$$

This would imply $\alpha= \pm 1$ and then $\pm 2^{p-1}=\sum_{r=0}^{\frac{1}{2}(p-1)}\left(\begin{array}{c}p \\ 2 r\end{array}\right)\left(-D \beta^{2}\right)^{r}$, with the lower sign rejected modulo $p$. Thus $2^{p-1}=\frac{1}{2}\left((1+\sqrt{1-x})^{p}+(1-\sqrt{1-x})^{p}\right)=f_{p}(x)$,

Received by the editors October 31, 2000.

2000 Mathematics Subject Classification. Primary 11R29; Secondary 11D61, 11B37, 11B39. 
TABLE 1.

\begin{tabular}{|c|c|c|c|c|c|}
\hline$n$ & $h /(n-2)$ & $n$ & $h /(n-2)$ & $n$ & $h /(n-2)$ \\
\hline 3 & 1 & 15 & 4 & 27 & 156 \\
\hline 4 & 1 & 16 & 8 & 28 & 384 \\
\hline 5 & 1 & 17 & 19 & 29 & 480 \\
\hline 6 & $1 / 4$ & 18 & 4 & 30 & 280 \\
\hline 7 & 1 & 19 & 15 & 31 & 685 \\
\hline 8 & 2 & 20 & 8 & 32 & 1408 \\
\hline 9 & 2 & 21 & 6 & 33 & 1776 \\
\hline 10 & 2 & 22 & 44 & 34 & 1982 \\
\hline 11 & 2 & 23 & 74 & 35 & 1728 \\
\hline 12 & 2 & 24 & 24 & 36 & 1792 \\
\hline 13 & 5 & 25 & 164 & 37 & 6108 \\
\hline 14 & 6 & 26 & 202 & & \\
\hline
\end{tabular}

say, where $x=1+D \beta^{2} \equiv 0(\bmod 8)$, and we show that this is impossible for any odd integer $p$, by showing that for each odd $k \geq 3$

$$
f_{k}(x) \equiv 2^{k-1}-k x \cdot 2^{k-3}\left(\bmod x \cdot 2^{k-2}\right) .
$$

Since $(1+\sqrt{1-x})^{2}+(1-\sqrt{1-x})^{2}=4-2 x$ and $(1+\sqrt{1-x})^{2}(1-\sqrt{1-x})^{2}=x^{2}$, we obtain the recurrence relation $f_{k+4}(x)=(4-2 x) f_{k+2}(x)-x^{2} f_{k}(x)$ with the values $f_{3}(x)=4-3 x$ and $f_{5}(x)=16-20 x+5 x^{2}$. Thus (11) holds for these values since $8 \mid x$, and we proceed to prove it by induction for larger $k$. If it holds for odd values $t$ and $t+2$, then

$$
\begin{aligned}
f_{t+4}(x)= & (4-2 x) f_{t+2}(x)-x^{2} f_{t}(x) \\
= & (4-2 x)\left(2^{t+1}-(t+2) x \cdot 2^{t-1}+A x \cdot 2^{t}\right) \\
& -x^{2}\left(2^{t-1}-t x \cdot 2^{t-3}+B x \cdot 2^{t-2}\right) \\
= & 2^{t+3}-(t+4) x \cdot 2^{t+1}+C x \cdot 2^{t+2},
\end{aligned}
$$

say, where $C=A+\frac{x}{4}(t+2)-\frac{1}{2} A x-\frac{1}{8} x+\frac{1}{32} t x^{2}-\frac{1}{16} B x^{2}$ is an integer.

Thus $\nu$ has no odd prime factor. Finally suppose that $2 \mid \nu$. Then we obtain that $\pm 2(1+a \sqrt{-D})=(\alpha+\beta \sqrt{-D})^{2}$, since now the unit \pm 1 can no longer be absorbed into the power. Then $\pm 2=\alpha^{2}-D \beta^{2}, \pm a=\alpha \beta$. But since $D \equiv 7(\bmod 8)$ we must reject the lower sign in the former, and then find

$$
2^{n}=1+D a^{2}=1+D \alpha^{2} \beta^{2}=\alpha^{4}-2 \alpha^{2}+1=\left(\alpha^{2}-1\right)^{2}
$$

and so $(\alpha+1)(\alpha-1)=2^{\frac{1}{2} n}$ whence for some integers $i>j, \alpha+1=2^{i}, \alpha-1=$ $2^{j}, 2=2^{i}-2^{j}$, yielding only $i=2, j=1, \alpha=3$, leading to $n=6$ and $D=7$ as required.

The author wishes to express his appreciation to the referee for providing the references, and for suggesting an improvement in the exposition.

A table showing the first few values of $h /(n-2)$ is given in Table 1 . 


\section{REFERENCES}

1. N. C. Ankeny and S. Chowla, On the divisibility of the class numbers of quadratic fields, Pacific J. Math. 5 (1955), 321-324. MR 19:18f

2. B. H. Gross and D. E. Rohrlich, Some results on the Mordell-Weil group of the Jacobian of the Fermat curve, Inventiones Math. 44 (1978), 201-224. MR 58:10911

Department of Mathematics, Royal Holloway University of London, Egham, Surrey TW20 0EX, United Kingdom

E-mail address: J.Cohn@rhul.ac.uk 